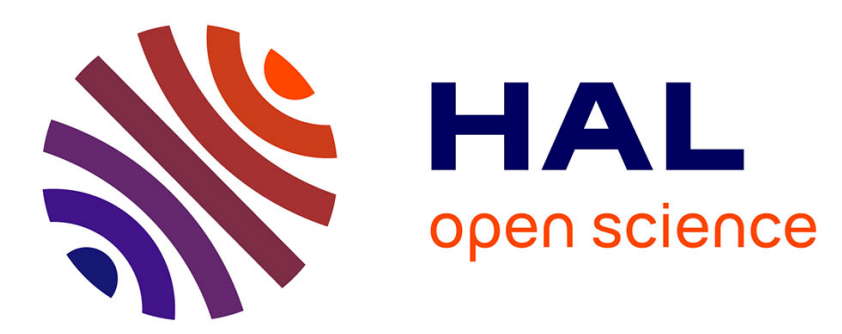

\title{
Caractérisation géométrique d'un condensat métallique sur diélectrique à l'approche du seuil de percolation
}

\author{
G. Desrousseaux, A. Carlan
}

\section{To cite this version:}

G. Desrousseaux, A. Carlan. Caractérisation géométrique d'un condensat métallique sur diélectrique à l'approche du seuil de percolation. Revue de Physique Appliquée, 1990, 25 (7), pp.753-761. 10.1051/rphysap:01990002507075300 . jpa-00246236

\section{HAL Id: jpa-00246236 https://hal.science/jpa-00246236}

Submitted on 1 Jan 1990

HAL is a multi-disciplinary open access archive for the deposit and dissemination of scientific research documents, whether they are published or not. The documents may come from teaching and research institutions in France or abroad, or from public or private research centers.
L'archive ouverte pluridisciplinaire HAL, est destinée au dépôt et à la diffusion de documents scientifiques de niveau recherche, publiés ou non, émanant des établissements d'enseignement et de recherche français ou étrangers, des laboratoires publics ou privés. 


\title{
Caractérisation géométrique d'un condensat métallique sur diélectrique à l'approche du seuil de percolation
}

\author{
G. Desrousseaux et A. Carlan \\ Laboratoire des Propriétés Physiques des Couches Minces, Université d'Aix-Marseille III, Faculté des \\ Sciences et Techniques de Saint-Jérôme, Avenue Escadrille Normandie-Niémen, 13397 Marseille Cedex 13, \\ France
}

(Reçu le 21 décembre 1989, révisé le 13 mars 1990, accepté le 29 mars 1990)

\begin{abstract}
Résumé. - A l'approche du seuil de percolation, les contours des îlots de dépôts métalliques discontinus sur substrat amorphe cessent d'être circulaires pour délimiter des espacements s'allongeant en forme de canaux. Nous proposons une modélisation de la répartition du métal dans de tels dépôts. L'utilisation de ce modèle nous permet de décrire quantitativement cette répartition en utilisant les seules mesures de la conductance de couche et de l'épaisseur massique du dépôt. Cette conversion de ces mesures électriques en termes de quantités géométriques suppose que le mécanisme prépondérant de la conduction électrique entre les agrégats est l'effet tunnel via le substrat.

Abstract. - At the approach of the percolation threshold, the outlines of islands of discontinuous metallic deposits on amorphous substrate, cease to be circular so as to delimite channels. We propound a modellisation of the metal repartition in these deposits. This modellisation enables us to quantitatively depict this repartition by only measuring the sheet conductance and the mass thickness of the deposit. This conversion of these electrical measures in terms of geometrical quantities supposes that the prevalent mechanism of electrical conduction between the islands is the tunnelling via their substrate.
\end{abstract}

\section{Introduction.}

Notre présent objectif est la mise au point d'une méthode permettant d'étendre, jusqu'au seuil de percolation, l'étude in situ de la géométrie d'un condensat métallique discontinu sur substrat diélectrique. En microscopie électronique par transmission (MET), on observe, à température ambiante, après une compétition plus ou moins durable entre nucléation et coalescence, une rapide prédominance de cette dernière [1]. La croissance de la couche métallique très mince atteint alors (Fig. 1a) son stade à haut degré de coalescence (HDC). Dans l'étape suivante (Fig. 1b), cette couche montre une répartition du métal condensé qui cesse d'être granulaire; de larges îlots $y$ délimitent dès lors des espacements uniformes présentant l'aspect de «canaux " qui débouchent sur des "carrefours". Malgré leur contour irrégulier, les îlots maintiennent ainsi le long de ces canaux un parallélisme de leurs bords en regard. Leur distance bord à bord $s$, s'identifiant à la largeur des canaux, n'évolue alors que très lentement $[2,3]$. L'analyse dimensionnelle de micrographies montre que la distribution des valeurs de $s$ est remarquablement étroite. En outre, au fur et à mesure de leur disparition, les canaux présentent une longueur $\ell_{\mathrm{c}}$ variant peu dans le plan du condensat. Cette uniformité $\mathrm{d}$ dépôt métallique résulte du processus d'élimination des canaux à l'approche du seuil de percolation. En effet, à ce stade d'évolution du dépôt, cette élimination s'effectue de plus en plus par obturation d'une extrêmité d'un canal puis raccourcissement de l'impasse ainsi formée dans l'amas que deux îlots constituent par coalescence statique (Fig. 1c). Dès l'apparition de canaux, les atomes incidents sont presque exclusivement consommés pour accroître la couverture métallique du substrat. L'accroissement de la hauteur $h_{\mathrm{i}}$ des îlots va ainsi en s'atténuant ; une éventuelle stabilisation de $h_{\mathrm{i}}$ ne peut survenir 


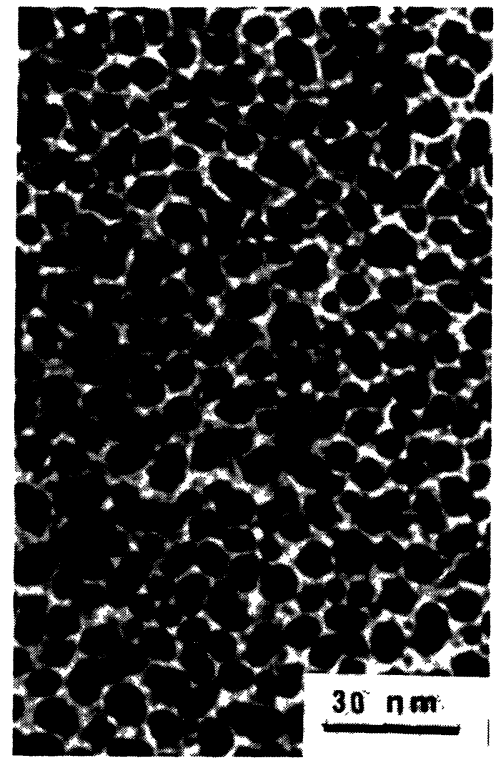

a)

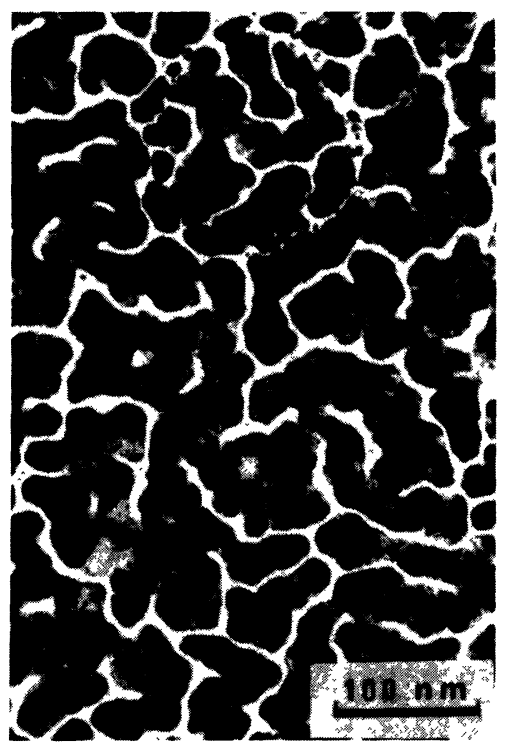

c)

qu'entre l'amorce de la percolation et le total recouvrement de la surface du substrat $[1,4-6]$. Nous ne pouvons donc postuler cette stabilisation durant l'évolution géométrique que nous nous proposons de décrire. La succession des étapes, précédant le seuil de percolation, apparaît en figure 2 .

Nous avons précédemment montré comment, à un instant $t$ de la formation sous ultravide d'un condensat métallique sur un diélectrique amorphe, les mesures couplées de l'épaisseur $e_{\mathrm{d}}$ et de la conductance de couche $C_{\mathrm{c}}$ permettent une description quantitative de la répartition du métal sur son substrat. Nous avions, pour cela, utilisé une modélisation du condensat granulaire demeurant réaliste durant la nucléation et la croissance tant que la coalescence reste modérée [7-10]. Lorsque survient

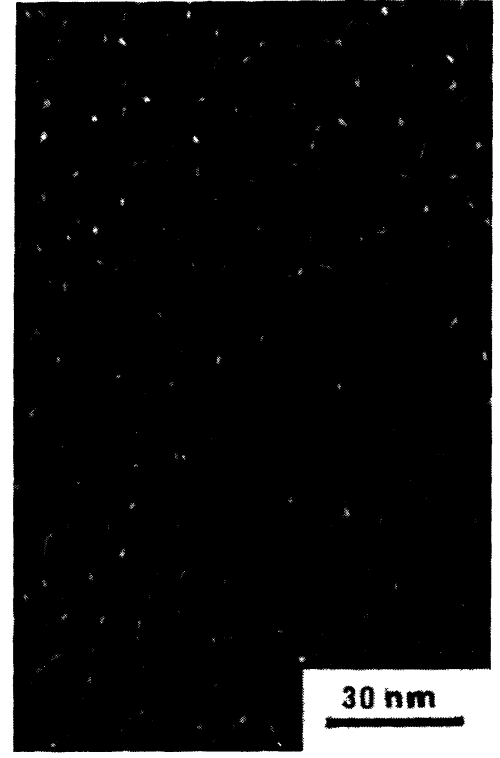

b)

Fig. 1. - a) Micrographie du système $\mathrm{Au} / \mathrm{MgO}$ noté $\mathrm{S} 1$; sa géométrie se situe en début de validité de cette modélisation. b) Micrographie du système $\mathrm{Au} / \mathrm{MgO}$ noté $\mathrm{S} 2$; le condensat d'or a atteint la géométrie pour laquelle la modélisation est bien adaptée. c) Micrographie d'un système $\mathrm{Au} / \mathrm{MgO}$ qui approche le seuil de percolation audelà duquel le modèle cesse d'être utilisable.

[a) Micrograph of $\mathrm{Au} / \mathrm{MgO}$ system labelled $\mathrm{S} 1$; its geometry shows the beginning of the suitability of the modelling. b) Micrograph of $\mathrm{Au} / \mathrm{MgO}$ system labelled $\mathrm{S} 2$; the condensate has reached the geometry for which the modelling is well suitable. c) Micrograph of $\mathrm{Au} / \mathrm{MgO}$ system wich approaches the threshold of percolation beyond wich the modelling ceases to be suitable.]

ce HDC, la précédente modélisation perd progressivement son réalisme au fur et à mesure que s'étend l'apparition des canaux. D'une géométrie à grains ellipsoïdaux, de taille et de distance à peu près constantes dans le plan de la couche, nous passons à des canaux dont la largeur et la longueur restent les seules à présenter une telle uniformité dans ce plan. Nous proposons, à présent, une adaptation de cette représentation de la répartition du métal sur son substrat en contrôlant par MET que la description ainsi obtenue reste statistiquement exacte jusqu'au seuil de percolation. Le critère de départ de cette remodélisation sera l'abaissement de la distance bord à bord $s$ entre les agrégats au niveau de la largeur de leur halo de capture $[6,11]$. Le calcul de la fraction $c$ de l'aire du substrat couverte par le 


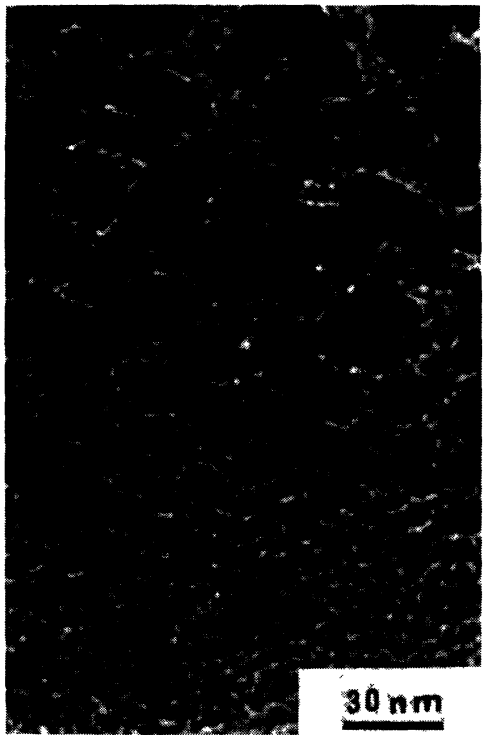

Fig. 2. - Cette micrographie montre les géométries des figures la et $1 \mathrm{~b}$ dans la succession des répartitions du métal depuis le stade granulaire jusqu'à l'apparition de canaux puis leur obturation.

[This micrograph shows the geometries $1 \mathrm{a}$ and $1 \mathrm{~b}$ in the sequence of metal repartitions from the granular stage till the appearance of channels next their obturation.]

métal permet de situer quantitativement [12-15] l'apparition du seuil de percolation au-delà duquel ce modèle est mis en défaut.

Pour contrôler le réalisme de cette nouvelle modélisation, nous avons à préparer simultanément deux ensembles dépôt/substrat, l'un destiné à des mesures de conduction, l'autre supporté par des grilles pour le contrôle en MET.

\section{Dispositif expérimental.}

Les condensations du diélectrique, servant de substrat, et du métal, constituant les îlots, sont successivement effectuées dans une enceinte à ultravide entièrement étuvable. Une pression résiduelle de l'ordre du $\mu \mathrm{Pa}$ est atteinte par pompage ionique.

Le métal $(\mathrm{Au})$ est évaporé à partir d'une cellule de Knudsen dont le flux atomique $q$ est préalablement mesuré au moyen d'une balance iézoélectri ue.

Les substrats sont des films de $\mathrm{MgO}$ préparés au moyen d'un canon à électrons. L'épaisseur $e_{\mathrm{s}} \mathrm{du}$ substrat est choisie de manière à obtenir le meilleur compromis possible entre une bonne continuité de la surface et une conductance de couche suffisamment faible devant celle du condensat métallique supporté.

Pour cette étude de la répartition du dépôt métallique discontinu sur son substrat, les condensations du diélectrique puis du métal sont effectuées successivement sur une lamelle de verre (Fig. 3) préalablement couverte par deux électrodes planes d'argent de forme rectangulaire (Fig. 3); les deux

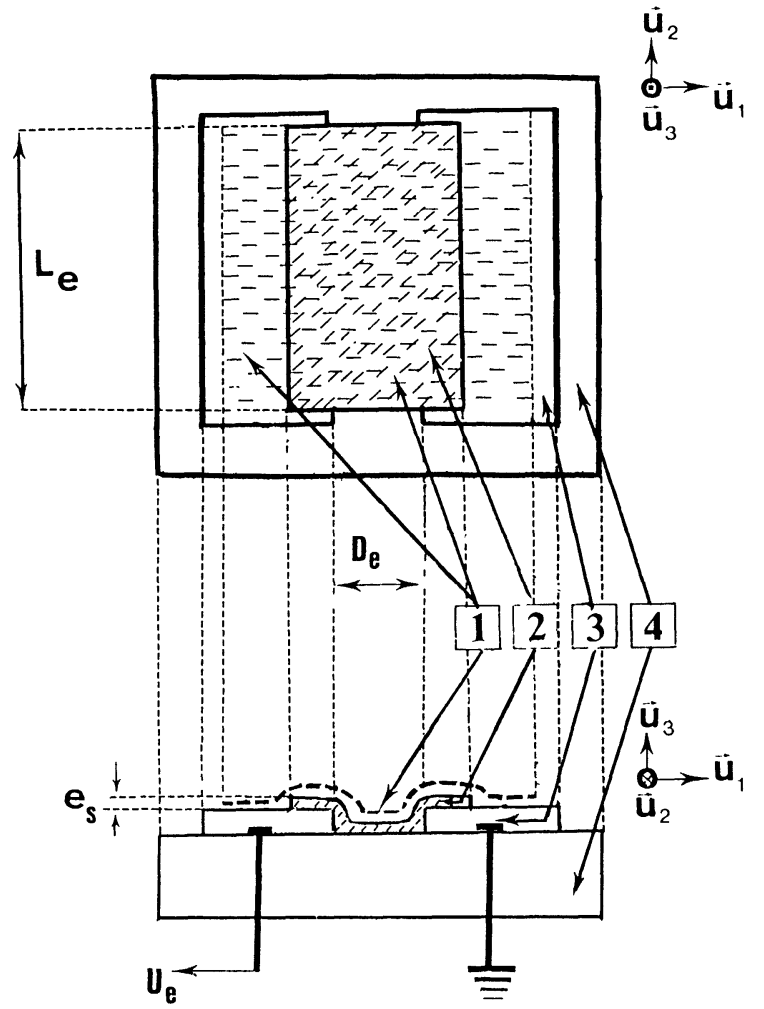

Fig. 3. - Support de verre (4), muni de ses électrodes planes d'argent déposées sous vide (3). En remontant, on distingue le film de $\mathrm{MgO}$ servant de substrat (2), le condensant d'or discontinu (1).

[Glass holder (4) with two evaporated silver contacts (3). It is successively covered with the $\mathrm{MgO}$ substrate film (2) and the discontinuous gold condensate.]

films minces les constituant ont une longueur $L_{\mathrm{e}}=20 \mathrm{~mm}$ et une distance $\mathrm{D}_{\mathrm{e}}=2 \mathrm{~mm}$. Une tension continue $U_{\mathrm{e}}=0,5 \mathrm{~V}$ est maintenue entre ces électrodes planes [7,9].

En fin de formation, le substrat est traversé par un courant d'intensité $I_{\mathrm{s}}$. Durant la condensation du métal, on observe à un instant $t$, un accroissement d'intensité $I_{\mathrm{d}}(t)$ correspondant à une épaisseur massique $e_{\mathrm{d}}(t)=q t$. Au stade de la condensation auquel apparaît une répartition du métal délimitant des canaux, le coefficient de collage est égal à l'unité. Nous n'aurons donc as à introduir entre le flux atomique incident et le nombre d'atomes condensés sur l'unité d'aire du substrat.

Avec ces notations, la conductance de couche s'exprime par

$$
C_{\mathrm{c}}=\left(I_{\mathrm{d}} / U_{\mathrm{e}}\right)\left(D_{\mathrm{e}} / L_{\mathrm{e}}\right)
$$

et la couverture atomique $n_{\mathrm{a}}$ du métal sur l'unité d'aire de son substrat par

$$
n_{\mathrm{a}}=e_{\mathrm{d}} / v^{*}
$$

$v^{*}$ désignant le volume atomique. 


\section{Modélisation et fondements théoriques.}

A ce stade de croissance auquel nous étudions ici l'évolution de la géométrie du condensat métallique, les îlots ont, dans le plan du substrat, des dimensions de l'ordre de $10 \mathrm{~nm}$. Leurs propriétés électroniques sont donc très proches de celles du métal massif [1620]. Ainsi au voisinage immédiat des interfaces électrodes/substrat et îlots/substrat, les lignes de champ sont normales à ces surfaces interfaciales tandis que, dans le reste du diélectrique, elles s'orientent parallèlement au support vers les îlots ou l'électrode à plus bas potentiel.

A basse température et faible champ électrique, le calcul montre que le courant circulant, dans le plan du support, entre les deux électrodes sous tension, est essentiellement attribuable à un effet tunnel entre îlots via le substrat $[15,21]$. Dans ces conditions, ce substrat constitue, entre ses interfaces avec deux îlots voisins, une superposition de «barrièrestunnel " dont la largeur est limitée inférieurement par $s$. Leur limite supérieure s'obtient en ajoutant à $s$ la dimension du plus étroit des deux îlots, en bordure du canal que le courant doit franchir normalement à sa ligne médiane.

Le courant circulant entre deux îlots voisins sera donc la somme des courants élémentaires allant d'un élément d'aire $\mathrm{d} S$ de l'interface, sous-jacente à un îlot en amont de la barrière, vers celui $\mathrm{d} S^{\prime}$ de l'interface voisine située en aval. En raison de l'élargissement rapide de la barrière minimale à l'abord des carrefours, nous pouvons considérer que l'essentiel de ce «courant-tunnel » circule perpendiculairement aux canaux. Nous obtiendrons donc une bonne approximation du courant entre îlots voisins (Fig. 4) en prenant pour $\mathrm{d} S$ et $\mathrm{d} S^{\prime}$ des bandes parallèles à la ligne médiane du canal, de longueur $\ell_{\mathrm{c}}$ et de largeur $\mathrm{d} x$, positionnées à la distance $x$ de cette ligne. Avec ces notations, les éléments d'aire s'expriment par

$$
\mathrm{d} S=\mathrm{d} S^{\prime}=\ell_{\mathrm{c}} \mathrm{d} x .
$$

Quelle que soit la courbure de la ligne médiane du canal mitoyen, on peut, en tout point de cette ligne, dans sa direction perpendiculaire, définir pour chacun des deux îlots voisins, une dimension $2 x_{M}-s$ égale à leur largeur dans cette direction.

Lorsque l'on s'éloigne de cette ligne médiane, les éléments $\mathrm{d} S$ et $\mathrm{d} S^{\prime}$ apportent au courant entre deux îlots voisins une contribution qui chute rapidement avec $x$. Nous pouvons donc choisir pour $2 x_{M}-s$ la largeur du plus étroit des îlots en regard. Dans le calcul du courant entre deux îlots voisins, $x_{M}$ sera pris comme la borne supérieure dans l'intégration, sur $x$, de la densité du courant d'effet tunnel via le substrat.

L'aire moyenne $A_{\mathrm{i}}$ des îlots peut être considérée comme la moyenne des produits de leurs dimensions,

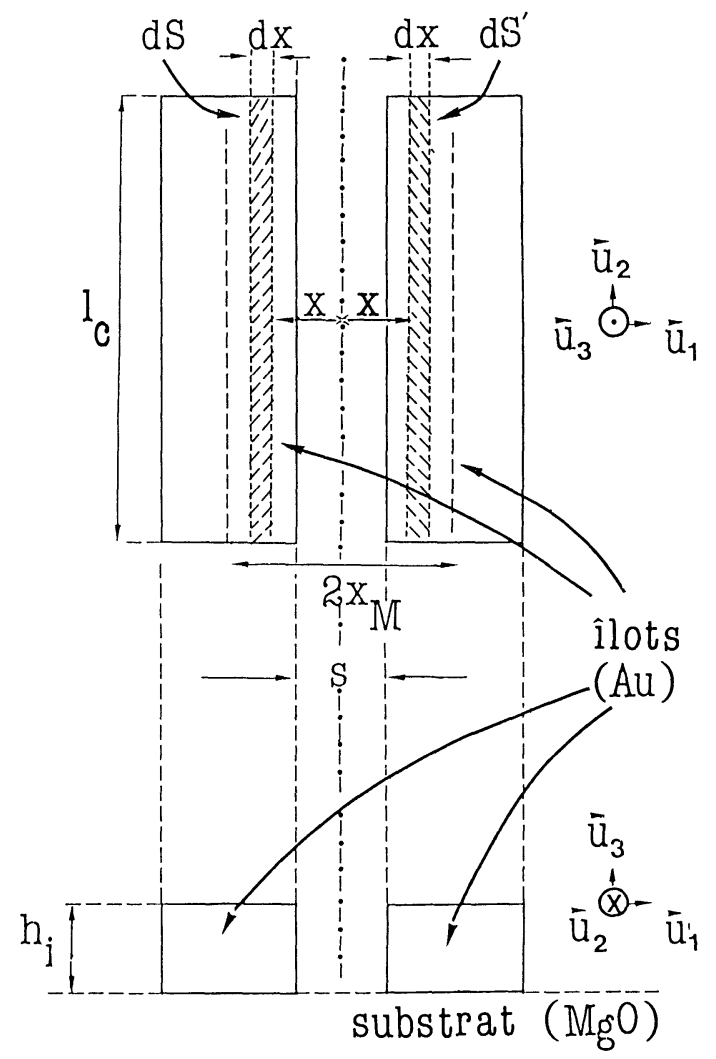

Fig. 4. - Représentation schématique de deux îlots voisins échangeant leurs charges interfaciales par effet tunnel via le substrat. d $S$ et $\mathrm{d} S^{\prime}$ désignent les éléments d'aire sur chacune des deux interfaces qui séparent les îlots de leur substrat.

[Schematic representation of two neighbouring islands which exchange their interface charges by tunnelling via the substrate. $\mathrm{d} S$ and $\mathrm{d} S^{\prime}$ label the area elements on each of two interfaces which separate the islands from their common substrate.]

l'une $\ell_{\mathrm{c}}$ parallèle aux bords du canal et l'autre $2 x_{\mathrm{M}}-s$ perpendiculaire. Nous noterons $z$ le rapport de ces deux dimensions et $n_{\mathrm{i}}$ la densité des îlots.

En désignant par $J(x)$ la densité de courant entre $\mathrm{d} S$ et $\mathrm{d} S^{\prime}$ à travers une barrière de largeur $2 x$, nous pouvons écrire que ce courant $I_{\mathrm{d}}$ circulant dans le dépôt entre les deux électrodes s'exprime par :

$$
I_{\mathrm{d}}=L_{\mathrm{e}}\left(n_{\mathrm{i}} / z\right)^{1 / 2} \ell_{\mathrm{c}} \int_{s / 2}^{x_{\mathrm{M}}} J(x) \mathrm{d} x
$$

Cette expression est écrite en admettant que l'ensemble des îlots offre $L_{\mathrm{e}}\left(n_{\mathrm{i}} / z\right)^{1 / 2}$ voies de conduction.

En substituant

$$
\ell_{\mathrm{c}}\left(2 x_{\mathrm{M}}-s\right)=A_{\mathrm{i}}
$$

et

$$
z\left(2 x_{\mathrm{M}}-s\right)=\ell_{\mathrm{c}}
$$


dans l'expression (4), on obtient:

$$
I_{\mathrm{d}}=L_{\mathrm{e}}\left(n_{\mathrm{i}} A_{\mathrm{i}}\right)^{1 / 2} \int_{s / 2}^{\left[\left(A_{\mathrm{i}} / z\right)^{1,2}+s\right] / 2} J(x) \mathrm{d} x .
$$

En introduisant la fraction de l'aire du substrat couverte par le métal

$$
c=n_{\mathrm{i}} A_{\mathrm{i}}
$$

et la distance bord à bord moyenne

$$
s=n_{\mathrm{i}}^{-1 / 2}-\left(c / n_{\mathrm{i}}\right)^{1 / 2}=n_{\mathrm{i}}^{-1 / 2}\left(1-c^{1 / 2}\right)
$$

on parvient finalement à l'équation

$$
c^{1 / 2} \int_{B_{\mathrm{i}}}^{B_{\mathrm{s}}} J(x) \mathrm{d} x=I_{\mathrm{d}} / L_{\mathrm{e}}
$$

où

$$
B_{\mathrm{i}}=n_{\mathrm{i}}^{-1 / 2}\left(1-c^{1 / 2}\right) / 2
$$

et

$$
B_{\mathrm{s}}=n_{\mathrm{i}}^{-1 / 2}\left[1-c^{1 / 2}\left(1-z^{-1 / 2}\right)\right] / 2
$$

sont respectivement les bornes inférieure et supérieure de l'intégrale.

Les barrières-tunnel élémentaires ont, toutes, pour hauteur l'écart énergétique $E_{\mathrm{c}}-E_{\mathrm{F}}$ que présente par rapport au niveau de Fermi le bas de la bande de conduction du diélectrique constituant le substrat $[19,20]$.

Pour calculer $J(x)$, nous utilisons la théorie de Hill [21] sous la formulation de Barr et Finney [22] avec $A=(8 \pi x / h)\left(2 m^{*}\right)^{1 / 2}$ et $B=A /\left[2\left(E_{\mathrm{c}}-E_{\mathrm{F}}\right)^{1 / 2}\right]$, notations de Simmons [23]. La tension entre îlots voisins est $V=U_{\mathrm{e}}\left(n_{\mathrm{i}}^{-1 / 2}\right) / D_{\mathrm{e}}$. L'énergie électrostatique d'activation $\delta E$ pour la conduction électronique est simplement la différence entre le travail à fournir contre la force image pour amener un électron d'un îlot à son voisin via le substrat et la variation de son énergie potentielle dans le champ local [15, 23, 24].

La densité du courant $J(x)$ s'écrit avec ces notations :

$$
J(x)=4 \pi m e h^{-3}\left\{\underset{2}{\left.\exp \left[-A\left(E_{\mathrm{c}}-E_{\mathrm{F}}\right)^{1 / 2}\right]\right\} \times}\right.
$$

avec

$$
\begin{aligned}
G & =G 1 / G 2+G 3 / G 4 \\
G 1 & =\exp (-B \delta E)-\exp (-B \mathrm{eV}) \\
G 2 & =1-\exp [(\delta E-\mathrm{eV}) / k T] \\
G 3 & =1-\exp [-B(\delta E+\mathrm{eV})] \\
G 4 & =1-\exp [(\delta E+\mathrm{eV}) / k T]
\end{aligned}
$$

Les relations (8) et (9) donnent immédiatement les valeurs moyennes de la distance bord à bord $s$ et de l'aire $A_{\mathrm{i}}$ des îlots, lorsque sont connus $c$ et $n_{\mathrm{i}}$.
Par contre pour exprimer l'épaisseur massique $e_{\mathrm{d}}=n_{\mathrm{i}} h_{\mathrm{i}} A_{\mathrm{i}}$, nous devons utiliser une expression empirique pour la fonction $b=h_{\mathrm{i}} / A_{\mathrm{i}}^{1 / 2}$ reliant $h_{\mathrm{i}}$ et $A_{\mathrm{i}}$.

A partir d'analyses dimensionnelles de micrographies, nous avons recherché graphiquement une relation entre $b$ et $e_{\mathrm{d}}$ pour les systèmes $\mathrm{Au} / \mathrm{MgO}$, $\mathrm{Pd} / \mathrm{MgO}, \mathrm{Au} / \mathrm{a}-\mathrm{C}$ et $\mathrm{Ag} / \mathrm{a}$ - C. Dans une représentation en double échelle logarithmique, les couples $\left(e_{\mathrm{d}}, b\right)$ s'alignent sur une portion rectiligne du tracé.

Pour le système $\mathrm{Au} / \mathrm{MgO}$, on obtient ainsi

$$
b=b^{*}\left(e_{\mathrm{d}} / e_{\mathrm{d}}^{*}\right)^{-1 / 8}
$$

où $b^{*}$ est l'ordonnée lue pour l'abscisse $e_{\mathrm{d}}^{*}$ sur une représentation de $\log b$ en fonction de $\log e_{\mathrm{d}}$. Le couple $\left(e_{\mathrm{d}}^{*}, b^{*}\right)$ repère le point à partir duquel un tracé rectiligne de pente $-1 / 8$ apparaît sur ce graphe. Ce coefficient $b$ est analogue à celui introduit par Pocza [1]. La M.E.T. montre que, pour cette épaisseur massique, les îlots métalliques commencent à délimiter des canaux. Pour $\mathrm{Au} / \mathrm{MgO}$, on trouve $b^{*}=0,31$ pour $e_{\mathrm{d}}^{*}=0,4 \mathrm{~nm}$; au-delà de $e_{\mathrm{d}}=2,5 \mathrm{~nm}$, les points cessent de s'aligner.

En introduisant $b$ dans l'expression de $e_{\mathrm{d}}$, il vient alors :

$$
n_{\mathrm{i}} A_{\mathrm{i}}^{3 / 2}=n_{\mathrm{i}}\left(c / n_{\mathrm{i}}\right)^{3 / 2}=n_{\mathrm{i}}^{-1 / 2} c^{3 / 2}=e_{\mathrm{d}} / b .
$$

Le système des relations (10) et (14) permet par itération de déterminer $n_{\mathrm{i}}$ et $c$ à partir des couples de mesures de $e_{\mathrm{d}}$ et $I_{\mathrm{d}}$. Dans ce domaine de répartition du métal qui marque l'approche du condensat vers la percolation, nous pouvons ainsi, durant sa formation, décrire quantitativement sa géométrie à partir du couple de mesures $\left(e_{\mathrm{d}}, I_{\mathrm{d}}\right)$. Cette description quantitative est faite en termes de concentration $n_{\mathrm{i}}$ des îlots, de fraction $c$ de l'aire du substrat couverte par le métal, de valeurs moyennes de la distance bord à bord $s$, de leur aire de projection $A_{\mathrm{i}}$ et de leur hauteur $h_{\mathrm{i}}$.

Le paramètre $z$ ne figure que dans l'expression de $B_{\mathrm{s}}$. Sa variation n'affecte pas sensiblement les quantités $c, n_{\mathrm{i}}$ et $s$. En effet, pour une telle géométrie du dépôt, l'intégrale de l'expression (10) dépend, en fait, fort peu de sa borne supérieure en raison de l'amortissement rapide avec $x$ de toutes les exponentielles présentes dans les expressions (11) et (12).

\section{Expérimentation et résultats.}

Pour contrôler le réalisme de notre modélisation, nous avons à présent à comparer les quantités géométriques déduites des mesures $e_{\mathrm{d}}$ et $I_{\mathrm{d}}$ avec celles que nous fournit l'analyse statistique des micrographies.

Nous donnons à cet effet dans le tableau I les résultats pour six systèmes $\mathrm{Au} / \mathrm{MgO}$. Leurs micrographies sont présentées en figures $1 \mathrm{a}, 1 \mathrm{~b}, 5,6,7$ et 
Tableau I. - Grandeurs décrivant la répartition de l'or d'un condensat discontinu sur MgO pour six systèmes. Les nombres soulignés sont des mesures sur les premières lignes, des moyennes statistiques sur les deuxièmes. Les données des calculs sont l'épaisseur et le courant traversant le dépôt dans son plan dans la méthode notée $\left(e_{\mathrm{d}}, I_{\mathrm{d}}\right)$, les moyennes statistiques relevées sur des micrographies dans celle notée M.E.T.

[Quantities which depict the gold repartition in the discontinuous condensate on $\mathrm{MgO}$ for six systems. The underline numbers are measurements on the first lines, mean statistical values on the second lines. The inputs of computations are the mass thickness and the planar current in the method labelled $\left(e_{\mathrm{d}}, I_{\mathrm{d}}\right)$, the mean statistical values deduced from micrograph in the method labelled MET.]

\begin{tabular}{|c|c|c|c|c|c|c|c|c|}
\hline Système & Méthode & $\begin{array}{c}e_{\mathrm{d}} \\
\mathrm{nm}\end{array}$ & $\begin{array}{r}I_{\mathrm{d}} \\
\mathrm{nA}\end{array}$ & $b$ & $\begin{array}{c}s \\
\mathrm{~nm}\end{array}$ & $\begin{array}{l}10^{-15} n_{\mathrm{i}} \\
\text { îlots } / \mathrm{m}^{2}\end{array}$ & $\begin{array}{c}c \\
\%\end{array}$ & $\begin{array}{c}A_{\mathrm{i}}^{1 / 2} \\
\mathrm{~nm}\end{array}$ \\
\hline - & - & - & - & - & - & - & - & - \\
\hline \multirow[t]{2}{*}{$\mathrm{S} 1$} & $\left(e_{\mathrm{d}}, I_{\mathrm{d}}\right)$ & $\underline{0,8}$ & $\underline{255}$ & 0,28 & 2,0 & 24,9 & 46 & 4,9 \\
\hline & M.E.T. & 1 & & 0,28 & $\underline{2}$ & $\underline{18}$ & 54 & 5,5 \\
\hline \multirow[t]{2}{*}{$\mathrm{S} 2$} & $\left(e_{\mathrm{d}}, I_{\mathrm{d}}\right)$ & $\underline{1,8}$ & $\underline{250}$ & 0,26 & 2,3 & 8,4 & 59 & 9,5 \\
\hline & M.E.T. & 1,8 & & 0,26 & $\underline{1,9}$ & $\underline{7,0}$ & 70 & 10 \\
\hline \multirow[t]{2}{*}{ S3 } & $\left(e_{\mathrm{d}}, I_{\mathrm{d}}\right)$ & $\underline{1,8}$ & 140 & 0,26 & 2,5 & 7,9 & 58 & 9,7 \\
\hline & M.E.T. & 1,82 & & 0,25 & $\underline{2,2}$ & $\underline{6,5}$ & 68 & 10,2 \\
\hline \multirow[t]{2}{*}{ S4 } & $\left(e_{\mathrm{d}}, I_{\mathrm{d}}\right)$ & $\underline{2,4}$ & 110 & 0,25 & 2,6 & 5,1 & 62 & 12,4 \\
\hline & M.E.T. & 2,52 & & 0,24 & $\underline{2,4}$ & 3,8 & 73 & 14 \\
\hline \multirow[t]{2}{*}{ S5 } & $\left(e_{\mathrm{d}}, I_{\mathrm{d}}\right)$ & $\underline{2,5}$ & 39 & 0,25 & 3,0 & 4,4 & 61 & 13,2 \\
\hline & M.E.T. & 2,5 & & 0,25 & $\underline{3,1}$ & $\underline{3,4}$ & 68 & 14 \\
\hline \multirow[t]{2}{*}{ S6 } & $\left(e_{\mathrm{d}}, I_{\mathrm{d}}\right)$ & $\underline{2,8}$ & 1200 & 0,24 & 1,9 & 5,1 & 69 & 13,2 \\
\hline & M.E.T. & 2,8 & & 0,24 & $\underline{1,9}$ & 3,6 & 78 & 15 \\
\hline
\end{tabular}

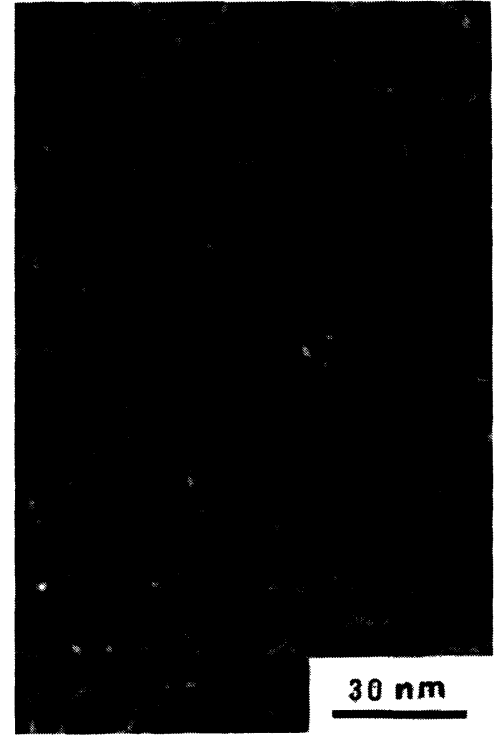

Fig. 5. - Micrographie du système $\mathrm{Au} / \mathrm{MgO}$ noté $\mathrm{S} 3$.

[Micrograph of the $\mathrm{Au} / \mathrm{MgO}$ labelled S3.]

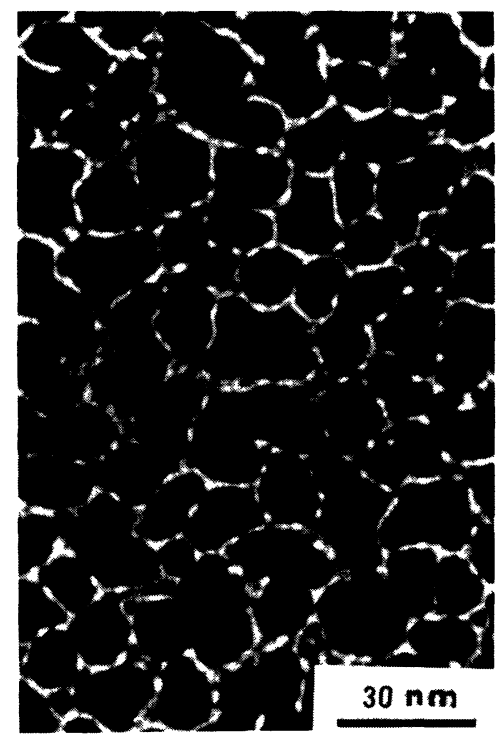

Fig. 6. - Micrographie du système $\mathrm{Au} / \mathrm{MgO}$ noté S4.

[Micrograph of the $\mathrm{Au} / \mathrm{MgO}$ labelled S4.] 


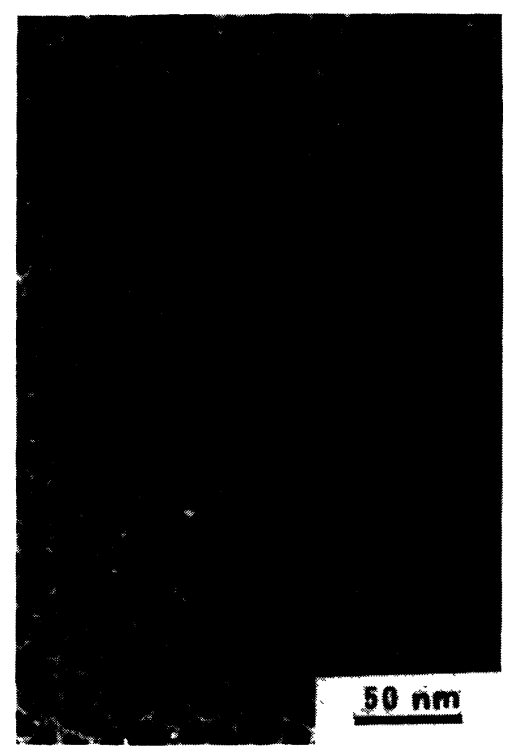

Fig. 7. - Micrographie du système $\mathrm{Au} / \mathrm{MgO}$ noté $\mathrm{S} 5$.

[Micrograph of the $\mathrm{Au} / \mathrm{MgO}$ labelled S5.]

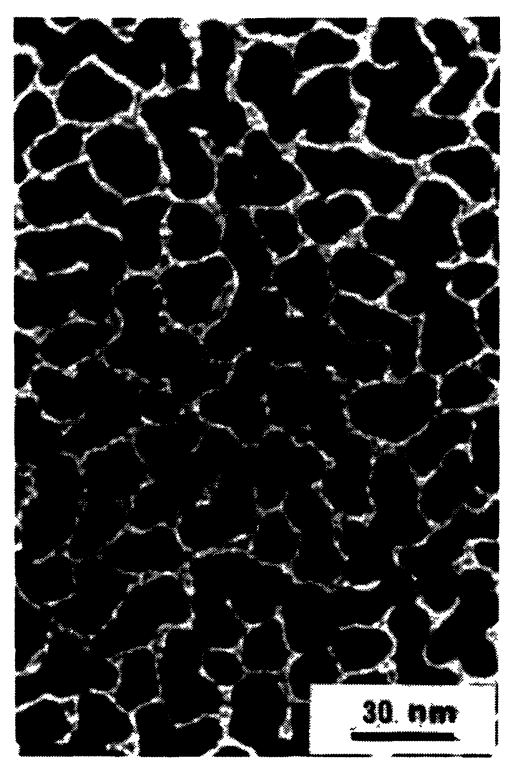

Fig. 8. - Micrographie du système $\mathrm{Au} / \mathrm{MgO}$ noté S6.

[Micrograph of the $\mathrm{Au} / \mathrm{MgO}$ labelled S6.]

8. Le système $\mathrm{S} 1$ (Fig. 1a) représente une limite inférieure pour le passage à cette nouvelle modélisation. Pour permettre d'apprécier la précision des mesures de courant et d'épaisseur massique, nous ajoutons dans le tableau II, en regard de la mention de chaque système, l'épaisseur $e_{\mathrm{s}}$ du substrat et l'intensité $I_{\mathrm{s}}$ du courant circulant dans son plan en fin de dépôt. D'autres informations sur les conditions de formation du substrat de $\mathrm{MgO}$ puis du condensat d'or complètent ce tableau II. Ce sont les vitesses $q_{\mathrm{s}}$ et $q$ des condensations successives de $\mathrm{MgO}$ et d'or et la température $T$ du système durant le dépôt du
Tableau II. - Conditions de préparation des six systèmes $\mathrm{Au} / \mathrm{MgO}$ étudiés dans le tableau I. On trouve de gauche à droite : l'épaisseur du substrat, le courant le traversant dans son plan avant condensation de l'or, sa vitesse de formation, celle de la condensation de l'or et la température du système durant cette condensation.

[Preparation conditions for the six $\mathrm{Au} / \mathrm{MgO}$ systems which are studied in table I. From left to right are given the substrate thickness $e_{\mathrm{s}}$, the planar current $I_{\mathrm{s}}$ before gold evaporating, the condensation rate $q_{\mathrm{s}}$ for $\mathrm{MgO}, q$ for the metal, the substrate temperature $T$ during the condensate formation.]

\begin{tabular}{cccccc} 
Système & $\begin{array}{c}e_{\mathrm{s}} \\
\mathrm{nm}\end{array}$ & $\begin{array}{c}I_{\mathrm{s}} \\
\mathrm{nA}\end{array}$ & $\begin{array}{c}q_{\mathrm{s}} \\
\mathrm{nm} / \mathrm{s}\end{array}$ & $\begin{array}{c}10^{-16} q \\
\mathrm{At} / \mathrm{m}^{2} / \mathrm{s}\end{array}$ & $\begin{array}{c}T \\
\mathrm{~K}\end{array}$ \\
\hline & - & - & - & - & - \\
S1 & 10 & 0,15 & 0,11 & 3,15 & 329 \\
S2 & 10 & 0,15 & 0,11 & 3,15 & 329 \\
S3 & 10 & 0,17 & 0,17 & 3,3 & 334 \\
S4 & 10 & 0,15 & 0,11 & 3,16 & 326 \\
S5 & 11 & 1 & 0,27 & 5,7 & 327 \\
S6 & 11 & 1 & 0,27 & 5,7 & 330
\end{tabular}

métal ; ces informations précisent les conditions de la répartition du métal. Elles seront utiles pour comprendre certaines accélérations de la coalescence que met en évidence le tableau I. La pression résiduelle de base est de l'ordre de $\mu \mathrm{Pa}$; sa remontée durant la condensation du métal n'excède pas un ordre de grandeur.

Ce tableau I montre un accord satisfaisant pour les quantités qui peuvent, dès l'apparition du H.D.C. dans la formation du condensats discontinu, décrire quantitativement sa géométrie jusqu'au seuil de percolation. On constate, en outre, que cet accord s'améliore lorsque la géométrie évolue vers ce seuil. Cette amélioration traduit celle du réalisme de ce nouveau modèle. Nous avons jugé nécessaire d'effectuer ce contrôle pour des dépôts métalliques ayant subi des effets plus ou moins prononcés de la coalescence. Pour faire ressortir de tels effets, nous présentons ces systèmes dans l'ordre de leur épaisseur croissante, et, pour une même épaisseur, dans l'ordre de leur conductance décroissante. La coalescence tend à augmenter $s$ et $A$. et corrélativement à diminuer $n_{\mathrm{i}}$. De tels changements dans la géométrie du dépôt se traduisent par une diminution de la conductance de couche pour une épaisseur massique donnée. C'est très exactement ce que montre ce tableau I. Ainsi les variations du courant, non seulement révèlent cette évolution géométrique du condensat métallique, mais en outre amplifient la mise en évidence de ces effets d'accélération de la coalescence. De cette manière, la méthode permet, sous ultravide et sous flux atomique, durant la formation du condensat, de suivre l'évolution de sa géométrie et d'en prévoir le sens. 


\section{Conclusion.}

Durant sa condensation sous ultravide un métal présente, avant la percolation, deux modes de répartition sur son substrat. Durant la compétition entre nucléation et coalescence, la géométrie du condensat apparaît sous forme de grains au contour régulier. Lorsque la coalescence devient prédominante les contours des agrégats se déforment pour délimiter entre leurs bords en regard des espacements uniformes que nous avons appelés " canaux ». Dans le premier mode, la répartition du métal peut être quantitativement décrite par le diamètre moyen des grains, leur densité et la fraction de l'aire du substrat qu'ils recouvrent. Dans le deuxième mode, la description quantitative de cette répartition sera plus réaliste si l'on utilise la densité des îlots, leur distance bord à bord et la fraction $1-c$ de l'aire du substrat qu'ils découvrent. Dans de précédents travaux [7-10] relatifs au premier mode, nous avons montré que cette description de la répartition du métal pouvait être déduite des mesures couplées de l'épaisseur massique $e_{\mathrm{d}}$ du condensat et de sa conductance de couche $C_{\mathrm{c}}$. Dans ce présent travail, nous montrons qu'en adaptant la modélisation du condensat, le second mode de répartition permet également une description quantitative de la dispersion du métal au sein du dépôt à partir de ces mêmes mesures $e_{\mathrm{d}}$ et $C_{\mathrm{c}}\left(\mathrm{ou} I_{\mathrm{d}}\right)$.

Le changement de modèle pose la question du choix du critère qui dicte le passage de la première modélisation à la seconde. Le franchissement de cette solution de continuité peut être réalisé en convenant que cette seconde modélisation sera adoptée dès que la distance bord à bord s'abaisse au niveau de $s_{\mathrm{c}}$. Cette distance $s_{\mathrm{c}}$ représente la largeur des halos de capture entourant les îlots $[6,11,25]$. Cette valeur critique, introduite dans la décroissance de $s$, n'est qu'une manière numérique d'écrire que l'apparition de ces canaux concrétise le recouvrement complet des halos de capture entourant les îlots.

\section{Bibliographie}

[1] PoczA J. F., Forming processes of directly observed vacuum-deposited thin films, Proc. 2nd Coll. on thin films, Ed. E. Hahn, (Vandenhoeck \& Ruprecht Gottingen) Budapest (1967) p. 93.

[2] KAzMersio L. L. and RACINE D. M., Growth environmental and electrical properties of ultrathin metal films, J. Appl. Phys. 46 (1975) 791.

[3] ANDERSON T., Electrical and structural properties of ultrathin gold films on glass, Ph. D. Thesis, Chalmers Univ. of Technology, Goteborg, Sweden (1976).

[4] Gadenne P., Beghdadi A. et Lafait J., Croissance de couches minces d'or sur verre. Structure fractale. Propriétés optiques et électriques à la percolation, J. Microsc. Spectrosc. Electron. 12 (1987) 477.

[5] Palevski A., Rappaport M. L., Kapitulnik A., Fried A. and Deutscher G., Hall coefficient and conduction in a $2 \mathrm{D}$ percolation system, $J$. Phys. Lett. France 45 (1984) L-367.

[6] Lewis B. and ANDERSON C., Nucleation and growth of thin fims (Academic Press Inc., London) 1978.

[7] Desrousseaux G., Trompette J., Faure R., SCHAFFAR H. et Dussaulcy J. P., Relation entre la granulométrie d'une couche métallique discontinue et les mesures de son épaisseur et de sa conductance, Thin Solid Films 98 (1982) 13.

[8] Desrousseaux G., Carlan A., Robrieux B., Trompette J., Schaffar H., Faure R. et DUSSAUlCY J. P., Conductance of metallic deposits on amorphous carbon during the nucleation and the growth, J. Non-Cryst. Solids 59-60 (1983) 529.
[9] Desrousseaux G., Carlan A., Robrieux B., Schaffar H., Trompette J., Dussaulcy J. P. et FAURE R., Caractérisation géométrique de dépôts métalliques discontinus sur film de carbone déduite des seules mesures d'épaisseur massique et de conductance de couche, J. Phys. Chem. Solids 46 (1985) 929.

[10] Robrieux B., Desrousseaux G., Renou A. and GILlET M., Morphology determination of small particles by electron microscopy and electrical conduction measurements, Zeitschrift für Physik, Atoms and Clusters 12 (1989) 23.

[11] Hamilton J. F., Preuss D. R. and Apai G. R., Nucleation and growth of vacuum, deposited metal aggregates studied by electron microscopy, Surf. Sci. 106 (1981) 146.

[12] ZALLEN R., The physics of amorphous solids (WileyInterscience Publication, John Wiley and Sons, Inc.) 1983.

[13] Romanowski W. and KePINSKi L., Structure and electrical conduction of discontinuous gold films evaporated in vacuum on carbon and silicon monoxide substrates, Thin Solid Films 65 (1980) 141.

[14] KePINSKi L., Dispersion and electrical resistance of discontinuous silver and cooper films on amorphous carbon and silicon oxide, Thin Solid Films 78 (1981) 133.

[15] Coutrs T. J., Electrical conduction in thin metal fims (Elsevier Scientific Publishing Company, Amsterdam) 1974.

[16] Halperin W. P., Quantum size effects in metal particles, Rev. Mod. Phys. 58 (1986) 533. 
[17] BAetzold R. C., Calculated properties of metal aggregates. II. Silver and palladium, J. Chem. Phys. 55 (1971) 4363 ; Molecular orbital description of silver clusters : Electronic structure, $J$. Chem. Phys. 68 (1978) 555, Electronic properties of metal clusters, Surf. Sci. 106 (1981) 243.

[18] a) BAetzold R. C. and MACK R. E., Electronic properties of metal clusters, J. Chem. Phys. 62 (1975) 1513 ;

b) Mason M. G. and Baetzold R. C., Esca and molecular orbital studies of small silver particles, J. Chem. Phys. 64 (1976) 271 ;

c) Baetzold R. C. and Hamilton J. F., Properties of small metal particles, Prog. Solid State Chem. 15 (1983) 1.

[19] Hill R. M., Electrical conduction in ultrathin metal films. I. Theorical, Proc. R. Soc. London, Ser. A 309 (1969) 377.
[20] BARR A. and Finney R. D., Tunnelling of electrons in discontinuous metal films, Thin Solid Films 24 (1974) 811.

[21] Simmons J. G., Generalized formula for the electrical tunnel effect between similar electrodes separated by a thin insulating film, J. Appl. Phys. 34 (1963) 1793.

[22] Abeles B., Sheng P., Coutts M. D. and ARie Y., Structural and electrical properties of granular films, Adv. Phys. 24 (1975) 407.

[23] Hamilton J. F. and Logel P. C., Thin Solid Films 23 (1974) 89 ; Vapor deposition of silver, gold and palladium on carbon and silicon dioxide in ion-pumped vacuum, Thin Solid Films 23 (19774) 89. 